\title{
Analysis of possible genetic risk factors contributing to development of childhood acute lymphoblastic leukaemia in the Latvian population
}

\author{
Madara Kreile ${ }^{1,2}$, Linda Piekuse ${ }^{1}$, Dmitrijs Rots ${ }^{1}$, Zane Dobele ${ }^{1}$, Zhanna Kovalova ${ }^{3}$, Baiba Lace ${ }^{4}$
}

\author{
${ }^{1}$ Scientific Laboratory of Molecular Genetics, Riga Stradiņš University, Riga, Latvia \\ ${ }^{2}$ Institute of Oncology, Riga Stradin, Š University, Riga, Latvia \\ ${ }^{3}$ Department of Hematology and Oncology, Children's Clinical University Hospital, \\ Riga, Latvia \\ ${ }^{4}$ Latvian Biomedical Research and Study Centre, Riga, Latvia
}

Submitted: 2 October 2014

Accepted: 10 November 2014

Arch Med Sci 2016; 12, 3: 479-485

DOI: 10.5114 /aoms.2016.59920

Copyright $\odot 2016$ Termedia \& Banach

\begin{abstract}
Introduction: Childhood acute lymphoblastic leukaemia (ALL) is a complex disease caused by a combination of genetic susceptibility and environmental exposure. Previous genome-wide association studies have reported several single nucleotide polymorphisms (SNPS) associated with the incidence of ALL. Several variations in genes encoding enzymes involved in carcinogenesis are suggested as being associated with an increased risk of ALL development. Material and methods: We enrolled 77 paediatric ALL patients and 122 healthy controls, and in addition parental DNA was also available for 45 probands. SNPs rs10821936 (ARID5B), rs4132601 (IKZF1), rs2239633 (CEBPE), rs3731217 (CDKN2A) and rs1800566 (NQO1) and the presence of GSTT1 and GSTM1 null variants were detected. For statistical analysis the hybrid method of two designs 'Haplin' was used as well as linkage disequilibrium for family-based association studies.

Results: We identified the SNP rs10821936 in the ARID5B gene as being statistically significantly associated with childhood ALL, especially if the C allele is in a homozygous state, relative risk (RR) 4.65, 95\% Cl: 2.03-10.6, $p=0.0006$. Statistically significant differences were not found in other SNPs. We found risk combinations including all five variations, the strongest association being found in a combination where all five genetic variants are in a homozygous state, CCTTTTTTCC, $p=0.032$.

Conclusions: The identified SNP rs10821936 could serve as a potential risk marker for childhood ALL development. Further studies in an independent population are needed for verification.
\end{abstract}

Key words: childhood acute lymphoblastic leukaemia, single nucleotide polymorphisms, ARID5B, IKZF1.

\section{Introduction}

Acute lymphoblastic leukaemia (ALL) is the most frequently occurring haematopoietic malignancy in children worldwide [1], with cure rates increasing from less than $10 \%$ in the 1960 s to over $80 \%$ nowadays [2]. Acute lymphoblastic leukaemia is a heterogeneous group of disorders that arise from the clonal proliferation of malignant, lymphoid cells in bone marrow, blood and other organs [3]. Approximately $75 \%$ of child-

\author{
Corresponding author: \\ Madara Kreile \\ Department of Hematology \\ and Oncology \\ Children's Clinical \\ University Hospital \\ 16 Dzirciema St \\ 1007 Riga, Latvia \\ Phone: 0037129679571 \\ E-mail: madara.kreile@rsu.lv
}


hood ALL cases harbour a recurring chromosomal alteration detectable by karyotyping, fluorescent in situ hybridization (FISH), or molecular biology techniques. Although rearrangements are important in initiating events in leukemogenesis and are widely used in diagnosis and risk stratification algorithms, they are insufficient to fully explain leukemogenesis [4]. However, even if the genetic markers of ALL can be detected in utero (i.e., ETV6RUNX1 gene), this does not inevitably lead to ALL [5]. The development of childhood B-cell lineage ALL involves (at least) 2 genetic events (hits), the first of which frequently arises prenatally [6]. The development of leukaemia might be explained by a combination of genetic susceptibility and environmental exposure during early development in fetal life and infancy [7].

The heritable basis of susceptibility to ALL is further supported by recent candidate gene and genome-wide association studies (GWAS), suggesting that co-inheritance of multiple germline variants may contribute to the risk of the disease. Five GWAS have been performed with populations of $3275 \mathrm{ALL}$ cases and 4817 healthy control individuals [8]. Four independent loci have been shown conclusively to be associated with ALL, and more specifically the B-cell precursor (BCP) ALL risk was associated with the particular SNPs located in 7p12.2 (IKZF1), 9p12 (CDKN2A/CDKN2B), $10 q 21.2(A R I D 5 B)$ and $14 q 11.2$ (CEBPE). While the risk of $A L L$ associated with these common variants is not insignificant (relative risk (RR) of $1.5-1.6)$, collectively they underscore $8-24 \%$ of the genetic variance in BCP-ALL risk [9]. The first GWAS performed by Trevino et al. evaluated 307944 germline single nucleotide polymorphisms (SNPs) and identified 18 SNPs whose allele frequency differed $\left(p<1 \times 10^{-5}\right)$ between a paediatric ALL population and non-ALL controls. The strongest association was shown by the SNP rs 10821936 located within intron 3 of the $A R I D 5 B$ gene [10]. AT-rich interactive domain 5B (ARID5B) belongs to the ARID family of transcription factors and plays an important role in the regulation of embryonic development and cell growth and differentiation [11]. However, the role of ARID5B in childhood ALL remains unknown, although alterations in gene function may contribute to an increased risk for this disease [12].

The second GWAS study showed that the IKAROS family zinc finger 1 (IKZF1) germline polymorphism rs4132601 is associated with inherited risk of paediatric ALL [13]. The product of the IKZFI gene is Ikaros, a sequence-specific DNA-binding protein with a zinc finger motif, which plays a role in immune homeostasis through the transcriptional regulation of the earliest stages of lymphocyte ontogeny and the differentiation by both gene transcriptional activation and repression [14]. The third association seen was at $14 q 11.2$, with rs2239633 in the vicinity of CEBPE, encoding the CCAAT/enhancer binding protein $\varepsilon$, a regulator of myelopoiesis [15]. Sherborne et al. reported an association between rs3731217 located in intron 1 of the gene CDKN2A (cyclin-dependent kinase inhibitor 2A) and childhood ALL [16].

One potentially important genetic pathway, besides the polymorphisms detected by GWAS that is suspected to play a role in the etiology of childhood ALL, is xenobiotic metabolism [17]. Polymorphisms in genes encoding xenobiotic-metabolizing enzymes are largely responsible for interindividual differences in the ability to activate and detoxify mutagenic/carcinogenic agents, and therefore may influence the susceptibility to cancer [18]. Environmental procarcinogens are activated by phase I enzymes, converting them into reactive intermediates, which are then detoxified by phase II enzymes including glutathione $S$ transferase (GST) [19]. Functional polymorphisms have been reported in the genes coding GSTs; $20-50 \%$ of individuals do not express the enzyme GSTT1 and/or GSTM1 due to homozygous deletion [20]. Individuals with the GSTT1 null and GSTM 1 null variant are unable to eliminate carcinogens effectively $[21,22]$. For example, they are unable to detoxify tobacco-related carcinogens by conjugating glutathione to facilitate their removal [23], and can therefore be a candidate risk factor for childhood ALL.

The NQO1 (NAD (P) H dehydrogenase quinone 1) protein protects cells against quinine-induced oxidative stress. There have been more than 93 polymorphisms identified, the most widely studied being NM_000903.2:C.559C>T or previously c.609C $>T$ (rs1800566). Homozygosity is associated with the loss of enzyme activity due to the instability of the protein product [24]. The heterozygous genotype showed a threefold decrease in enzymatic activity compared with the wild type allele [25]. NQO1 has been described as an anticancer enzyme because it plays a protective role in cancerogenesis by modifying internal exposure to bioactivated carcinogens [26], and in the case of a defective enzyme it might play a role in cancerogenesis.

Because of the possible roles of polymorphisms rs10821936 (ARID5B), rs4132601 (IKZF1), rs2239633 (CEBPE), rs3731217 (CDKN2A) - markers which showed the strongest association from GWAS data - and rs1800566 (NQO1), and null genotypes of genes GSTT1 and GSTM1, we performed case control and case-parent triad studies to investigate the association between these genetic markers and childhood ALL susceptibility in the Latvian population. 


\section{Material and methods}

\section{Study subjects}

Our cohort consisted of 76 patients with childhood B-cell progenitor cell ALL in complete remission, and 121 age- and gender-adjusted healthy controls. In addition, parental DNA was available for 45 probands. Out of 76 patients, 41 were male and 35 were female. Patients were diagnosed with ALL between 2005 and 2014, aged 1-18 years at that time. According to the data of the Latvian cancer patient registry, at the time of the study 85 patients with a diagnosis of ALL were alive (8 of them refused to take part in this study and 1 patient was not in full remission); therefore our study covered $89.4 \%$ of all patients. Parents signed an informed consent form, and the study was approved by the Central Medical Ethics committee of Latvia.

\section{Genotyping}

Peripheral blood from the patients and their biological parents and controls was used for DNA extraction. DNA isolation from white blood cells was performed using the standard phenol/chloroform method [27].

Genotyping of polymorphisms rs4132601, rs2239633, rs3731217, and rs1800566 was performed by polymerase chain reaction - restriction fragment length polymorphism (PCR-RFLP) assay; primer sequences and restriction enzymes are shown in Table I. Genotype of the polymorphism rs10821936 was detected by Sanger sequencing; for the initial PCR we used external primers (F: 5'-CAGTGAGAGCGAGACTGCAC-3'; R: 5'-TCCTGGCAATGTTTTTCACA-3'), while for the second amplification an internal primer was used (F: 5'-ACACCACCCCACATCTCAAT-3'). The direct sequencing of PCR products was performed using ABI Prism BigDye Terminator v3.1 Cycle sequencing kit (Applied Biosystems, Foster city, USA) using the manufacturer's protocol.
Multiplex PCR was performed to detect the presence or absence of GSTT1 and GSTM1 null genotypes [28].

\section{Statistical analysis}

Statistical analysis using the hybrid method for the case-control study and case-parent study was performed using the R program package "Haplin" [29]. To analyse case-parent trios the family-based association test (FBAT) by the transmission distortion test (TDT) and the haplotype-based TDT were performed, as implemented in the software PLINK 1.07 [30]. To separately analyse the case-control model the software JavaStat (available online) was used, the Fisher exact test was used for statistical analysis, and a $p$-value $<0.05$ was considered as statistically significant.

\section{Results}

All five analysed polymorphisms, rs10821936 (ARID5B), rs4132601 (IKZF1), rs2239633 (CEBPE), rs3731217 (CDKN2A) and rs1800566 (NQO1), were in Hardy-Weinberg equilibrium.

Linkage and case-control studies showed a significant association between ALL and rs10821936, which maps to intron 3 of the ARID5B gene. We did not find any significant association with rs4132601, which maps within the 3' UTR region of IKZF1, as well as rs2239633 in the locus of the CEBPE gene. When a hybrid statistical method (results shown in Table II) was used, statistical significance was more convincing than statistical analysis of separate association studies. The results of the family-based association study (TDT) are shown in Table III. The results of statistical analysis for case-control analysis are shown in Table IV. Separately analysing both designs showed the same results that the $C$ allele of rs10821936 increases the risk of ALL, especially in a homozygous state.

Using the hybrid statistical method we also identified some risk-increasing combinations for

Table I. Polymerase chain reaction primer sequences and restriction enzymes

\begin{tabular}{|c|c|c|c|}
\hline Polymorphism & Primer sequence & $\begin{array}{c}\text { Annealing } \\
\text { temperature }\left[{ }^{\circ} \mathrm{C}\right]\end{array}$ & Restriction enzyme \\
\hline \multirow[t]{2}{*}{ rs4132601 } & Forward: 5'-TCATGGATTTCTCTGCTCACA-3' & \multirow[t]{2}{*}{59} & \multirow{2}{*}{$\begin{array}{c}\text { Mbol (Thermo Fisher Scientific, } \\
\text { Waltham, MA, USA) }\end{array}$} \\
\hline & Reverse: 5'-TTCTGGTTTGAGCTTTGTTTGA-3' & & \\
\hline \multirow[t]{2}{*}{ rs2239633 } & Forward: 5'-GGGCTGGAAACGCACTAATA-3' & \multirow[t]{2}{*}{60} & \multirow{2}{*}{$\begin{array}{c}\text { Bsh1236l (Thermo Fisher } \\
\text { Scientific, Waltham, MA, USA) }\end{array}$} \\
\hline & Reverse: 5'-CСАCTCTGAGCAACCTCCTC-3' & & \\
\hline \multirow[t]{2}{*}{ rs3731217 } & Forward: 5'-CATGTAGATGGTGCCCCTGA-3' & \multirow[t]{2}{*}{55} & \multirow{2}{*}{$\begin{array}{c}\text { Mval (Thermo Fisher Scientific, } \\
\text { Waltham, MA, USA) }\end{array}$} \\
\hline & Reverse: 5'-CСССТСТСAАATATGCTGTCC-3' & & \\
\hline \multirow[t]{2}{*}{ rs1800566 } & Forward: 5'-TGAGAAGCCCAGACCAACTT-3' & \multirow[t]{2}{*}{60} & \multirow{2}{*}{$\begin{array}{c}\text { Hinfl (Thermo Fisher Scientific, } \\
\text { Waltham, MA, USA) }\end{array}$} \\
\hline & Reverse: 5'-TCTCCAGGCGTTTCTTCCAT-3' & & \\
\hline
\end{tabular}


Madara Kreile, Linda Piekuse, Dmitrijs Rots, Zane Dobele, Zhanna Kovalova, Baiba Lace

Table II. Hybrid analysis of SNP alleles in family trios and case-controls

\begin{tabular}{|lccccc|}
\hline Polymorphism & MAF & Dose & RR & $95 \% \mathrm{Cl}$ & $P$-value \\
\hline rs10821936 & $\mathrm{C}$ & Single & 1.3 & $(0.69-2.2)$ & 0.44 \\
\cline { 3 - 5 } & & Double & 4.65 & $(2.03-10.6)$ & 0.0006 \\
\hline rs4132601 & $\mathrm{G}$ & Single & 1.03 & $(0.58-1.78)$ & 0.92 \\
\cline { 3 - 5 } & & Double & 1.36 & $(0.54-3.3)$ & 0.5 \\
\hline rs2239633 & $\mathrm{T}$ & Single & 0.91 & $(0.52-1.63)$ & 0.76 \\
\cline { 3 - 5 } & & Double & 0.74 & $(0.33-1.65)$ & 0.47 \\
\hline rs3731217 & $\mathrm{G}$ & Single & 0.77 & $(0.37-1.65)$ & 0.5 \\
\cline { 3 - 5 } & & Double & - & - & 0.7 \\
\hline rs1800566 & T & Single & 0.94 & $(0.53-1.66)$ & 0.33 \\
\cline { 2 - 5 } & & Double & 0.57 & $(0.05-4.5)$ & \\
\hline
\end{tabular}

Table III. Family-based association study TDT test

\begin{tabular}{|lcc|}
\hline Polymorphism & OR $(95 \% \mathrm{Cl})$ & $P$-value \\
\hline rs10821936 & $2.07(1.07-4.03)$ & 0.027 \\
\hline rs4132601 & $0.73(0.4-1.32)$ & 0.3 \\
\hline rs2239633 & $0.62(0.35-1.12)$ & 0.11 \\
\hline rs3731217 & $1(0.32-3.1)$ & 1 \\
\hline rs1800566 & $0.8(0.38-1.67)$ & 0.68 \\
\hline
\end{tabular}

previously described SNPS (SNP order in genotype: rs10821936, rs4132601, rs2239633, rs3731217 and rs1800566); genotype CCTTCCTTCC was sta- tistically significantly more frequently observed in affected children $(p=0.046)$. Similar results were obtained for genotypes CCGGCCTTCC ( $p=0.049)$ and CCTTTTTTCC $(p=0.032)$.

The GSTT1 and GSTM1 null allele detection method has a limitation in that this method only detects the presence of the null genotype in a homozygous state. For statistical analysis we could only compare the frequency of the null genotype versus the non-null genotype. There was no statistically significant association between the risk of childhood acute leukaemia and polymorphisms of GSTM 1 and GSTT1. The GSTT1 null variant was observed in $27.6 \%$ of cases and in $18 \%$ of controls

Table IV. Frequency of SNPs in the patient and control groups

\begin{tabular}{|c|c|c|c|c|c|}
\hline Polymorphism & Allele & ALL cases & Controls & OR $(95 \% \mathrm{Cl})$ & $P$-value \\
\hline \multirow[t]{3}{*}{ rs10821936 } & CC & 14 (18.7\%) & $9(7.5 \%)$ & $2.83(1.1-7.59)$ & 0.02 \\
\hline & $\mathrm{CC}+\mathrm{CT}$ & $38(50.6 \%)$ & 40 (33.3\%) & $1.49(0.8-2.78)$ & 0.19 \\
\hline & C & 52 (34.6\%) & 71 (29.6\%) & $1.67(1.1-2.67)$ & 0.028 \\
\hline \multirow[t]{3}{*}{ rs4132601 } & GG & $8(10.6 \%)$ & $7(6.1 \%)$ & $1.83(0.56-5.9)$ & 0.28 \\
\hline & $G G+G T$ & $40(53.3 \%)$ & $51(44.7 \%)$ & $1.41(0.75-2.65)$ & 0.3 \\
\hline & G & $48(32 \%)$ & $58(25.4 \%)$ & $1.38(0.85-2.23)$ & 0.2 \\
\hline \multirow[t]{3}{*}{ rs2239633 } & TT & $13(17.1 \%)$ & $23(19.1 \%)$ & $0.87(0.38-1.96)$ & 0.85 \\
\hline & $\mathrm{TT}+\mathrm{CT}$ & $51(67.1 \%)$ & 75 (62.5\%) & $0.84(0.43-1.64)$ & 0.63 \\
\hline & $\mathrm{T}$ & $64(42.1 \%)$ & $108(45 \%)$ & $0.89(0.58-1.37)$ & 0.6 \\
\hline \multirow[t]{2}{*}{ rs3731217 } & GT & $12(16.2 \%)$ & $26(21.8 \%)$ & $0.69(0.3-1.56)$ & 0.36 \\
\hline & G & $12(8.1 \%)$ & $26(10.9 \%)$ & $0.72(0.33-1.55)$ & 0.48 \\
\hline \multirow[t]{3}{*}{ rs1800566 } & TT & $1(1.3 \%)$ & $5(4.1 \%)$ & $0.31(0.01-2.8)$ & 0.41 \\
\hline & $\mathrm{TT}+\mathrm{CT}$ & $22(28.9 \%)$ & $42(30.6 \%)$ & $0.82(0.42-1.58)$ & 0.54 \\
\hline & $T$ & 2 & 10 & $0.78(0.44-1.38)$ & 0.42 \\
\hline
\end{tabular}


$(p=0.16, \mathrm{OR}=1.69,95 \% \mathrm{Cl}: 0.8-3.53)$, and the GSTM null variant was observed in $55.8 \%$ of cases and in $47.9 \%$ of controls ( $p=0.3, \mathrm{OR}=1.37$, $95 \% \mathrm{Cl}: 0.74-2.54)$.

\section{Discussion}

Acute lymphoblastic leukaemia is a multifactorial disease influenced by genetic and environmental factors. In this study we investigated whether SNPs which were previously reported in GWAS studies influence the risk of ALL in the Latvian population. We chose the most studied cases, because Xu et al. hypothesized that the genetic predisposition to ALL might be largely mediated by the robust effects of a modest number of key genes, rather than the cumulative effect of tens of thousands of variants with small effects [31]. We also studied the influence of xenobiotic metabolism involved genes as a possible risk factor. Although our cohort covered $89.4 \%$ of all patients, an important limitation of this study was the sample size. This may have influenced our ability to detect small associations. In this study we were able to replicate the results from previous studies, showing that rs10821936 increases the susceptibility to childhood ALL. As is shown in the literature, this SNP has a strong association with childhood ALL [32, 33]. In our study we used the hybrid method to improve statistical significance and join two designs, and statistical analysis showed a stronger association than the designs separately. Analysis of our data showed that individuals with genotype CC have a statistically significantly increased risk of development of ALL, whereas statistical significance was lost if the individual was heterozygous. It is thought that gene variants have additive effects; someone inheriting one copy of a variant will have a roughly $50 \%$ increased risk, whereas someone who inherits all four variants in homozygous form would have a 10-fold increased risk [34].

In our study, rs4132601 in IKZF1 and rs2239633 in CEBPE did not seem to affect the susceptibility to childhood ALL, although an association was found in previous GWAS studies [13] using data from Asian colleagues Wang et al. in the Chinese population, and Lin et al. in Taiwanese children. They have published data where these polymorphisms do not show any significant difference between cases and controls [11,35]. Moreover, Wang et al. reported results of a cohort which consisted of 570 individuals, so small sample size could not be a limitation of that study. Vijayakrishnan et al. reported on the racial differences between risk allele frequency in Asians and Caucasians [36]. As we belong to the European population our results should be similar to other European countries, but the only available published data are from Poland, Pastorczak et al., who only iden- tified rs4132601 as a possible risk variant, and they could not replicate the reported associations with rs2239633 or rs3731217, nor could we [37]. Our results could be explained by the limited sample size. Although we are located close to Poland geographically, our ethnic origin is not as closely related as shown in a paper published by Nelis et al. [38]. To reach more reliable conclusions, further investigations closer to our gene pool are needed. However, Healy et al., from Canada, in a group of 284 childhood ALL patients, also were unable to replicate the reported associations with IKZF1 and CEBPE. Their group explained the lack of replication by the limited power of their study to detect loci with weaker effects [39]. In our study we were unable to find statistically significant differences between the GSTT1 and GSTM 1 null variants and the risk of childhood ALL. As reported in the literature, based on a meta-analysis performed by Xu et al. including 2424 cases, the GSTT1 null variant is strongly associated with the risk of childhood leukaemia in Asians, but there is no association in Caucasians [21]. Similar results about the GSTM1 null variant were reported in a meta-analysis by Tang et al., who found an overall association with ALL that was lost in Caucasians after stratification by ethnic groups [40], which might explain the lack of statistical significance in our study. Similarly, we could not find any association with another polymorphism involved in xenobiotic metabolism.

Although the meta-analysis performed by Han et al. showed an association with polymorphism 609 C>T ( $r$ 1800566), especially in a Caucasian population, they report that there is no association between NQO1 polymorphism and childhood leukaemia [41]. These results showed a similarity with ours, especially taking into consideration that only 18 out of 77 children in our ALL cohort were older than 10 at the time of the ALL diagnosis. We might hypothesize that our cohort was too young to observe the possible impact of polymorphisms involved in xenobiotic metabolism.

In conclusion, overall, the ARID5B SNP rs 10821936 has a significant association with the risk of childhood ALL - this polymorphism could serve as a genetic susceptibility marker for ALL. The cohort should be enlarged to estimate the real effect of other SNPS, or if Estonian and Lithuanian samples could be available to validate the effect of these SNPs in neighbouring populations.

\section{Conflict of interest}

The authors declare no conflict of interest.

\section{References}

1. Lautner-Csorba O, Gezsi A, Erdelyi DJ, et al. Roles of genetic polymorphisms in the folate pathway in childhood 
acute lymphoblastic leukemia evaluated by Bayesian relevance and effect size analysis. PLoS One 2013; 8: e69843.

2. Yang JJ, Cheng C, Devidas M, et al. Genome-wide association study identifies germline polymorphisms associated with relapse of childhood acute lymphoblastic leukemia. Blood 2012; 20: 4197-204.

3. Zhai X, Wang $H$, Zhu X, et al. Gene polymorphisms of $A B C$ transporters are associated with clinical outcomes in children with acute lymphoblastic leukemia. Arch Med Sci 2012; 8: 659-71.

4. Mullighan CG. The molecular genetic makeup of acute lymphoblastic leukemia. Hematology Am Soc Hemato Educ Program 2012; 2012: 389-96.

5. Peyrouze P, Guihard S, Grardel N, et al. Genetic polymorphisms in ARID5B, CEBPE, IKZF1 and CDKN2A in relation with risk of acute lymphoblastic leukaemia in adults a Group for Research on Adult Acute Lymphoblastic Leukaemia (GRAALL) study. Br J Haematol 2012; 5: 599-602.

6. Lausten-Thomsen U, Madsen HO, Vestergaard TR, Hjalgrim H, Nersting J, Schmiegelow K. Prevalence of $\mathrm{t}(12 ; 21)[$ ETV6-RUNX1]-positive cells in healthy neonates. Blood 2011; 1: 186-9.

7. Healy J, Belanger $H$, Beaulieu P, Lariviere M, Labuda D, Sinnett D. Promoter SNPs in G1/S checkpoint regulators and their impact on the susceptibility to childhood leukemia. Blood 2007; 2: 683-92.

8. Offenmuller S, Ravindranath Y, Goyette G, et al. Focused screening of a panel of cancer-related genetic polymorphisms reveals new susceptibility loci for pediatric acute lymphoblastic leukemia. Pediatr Blood Cancer 2014; 8: 1411-5.

9. Enciso-Mora V, Hosking FJ, Sheridan E, et al. Common genetic variation contributes significantly to the risk of childhood B-cell precursor acute lymphoblastic leukemia. Leukemia 2012; 10: 2212-5.

10. Trevino LR, Yang W, French D, et al. Germline genomic variants associated with childhood acute lymphoblastic leukemia. Nat Genet 2009; 9: 1001-5.

11. Lin CY, Li MJ, Chang JG, et al. High-resolution melting analyses for genetic variants in ARID5B and IKZF1 with childhood acute lymphoblastic leukemia susceptibility loci in Taiwan. Blood Cells Mol Dis 2014; 2-3: 140-5.

12. Gutierrez-Camino A, Lopez-Lopez E, Martin-Guerrero I, et al. Intron 3 of the ARID5B gene: a hot spot for acute lymphoblastic leukemia susceptibility. J Cancer Res Clin Oncol 2013; 11: 1879-86.

13. Papaemmanuil E, Hosking FJ, Vijayakrishnan J, et al. Loc on $7 p 12.2,10 q 21.2$ and $14 q 11.2$ are associated with risk of childhood acute lymphoblastic leukemia. Nat Genet 2009; 9: 1006-10.

14. Qazi, S, Ma H, Uckun FM. Absence of genomic deletions in pediatric B-precursor acute lymphoblastic leukemia Int J Mol Med Sci 2013; 9: 72-82.

15. Prasad RB, Hosking FJ, Vijayakrishnan J, et al. Verification of the susceptibility loci on 7p12.2, 10q21.2, and $14 q 11.2$ in precursor B-cell acute lymphoblastic leukemia of childhood. Blood 2010; 9: 1765-7.

16. Sherborne AL, Hosking FJ, Prasad RB, et al. Variation in CDKN2A at 9p21.3 influences childhood acute lymphoblastic leukemia risk. Nat Genet 2010; 6: 492-4.

17. Nousome D, Lupo PJ, Okcu MF, Scheurer ME. Maternal and offspring xenobiotic metabolism haplotypes and the risk of childhood acute lymphoblastic leukemia. Leuk Res 2013; 5: 531-5.

18. Huang GZ, Shan W, Zeng L, Huang LG. The GSTP1 A1578G polymorphism and the risk of childhood acute lymphoblastic leukemia: results from an updated meta-analysis. Genet Mol Res 2013; 3: 2481-91.

19. Chauhan PS, Ihsan R, Mishra AK, et al. High order interactions of xenobiotic metabolizing genes and P53 codon 72 polymorphisms in acute leukemia. Environ Mol Mutagen 2012; 8: 619-30

20. Dunna NR, Vure S, Sailaja K, et al. Deletion of GSTM1 and $\mathrm{T} 1$ genes as a risk factor for development of acute leukemia. Asian Pac J Cancer Prev 2013; 4: 2221-4.

21. Xu LY, Cao LF. GSTT1 genetic polymorphism and susceptibility to childhood acute lymphoblastic leukemia: a meta-analysis. Tumour Biol 2014; 2: 1433-7.

22. Liu D, Liu Y, Ran L, Shang H, Li D. GSTT1 and GSTM1 polymorphisms and prostate cancer risk in Asians: a systematic review and meta-analysis. Tumour Biol 2013; 5: 2539-44.

23. Amer MA, Ghattas MH, Abo-Elmatty DM, Abou-El-Ela SH. Evaluation of glutathione S-transferase $\mathrm{P} 1$ genetic variants affecting type- 2 diabetes susceptibility and glycemic control. Arch Med Sci 2012; 8: 631-6.

24. Ouerhani S, Cherif N, Bahri I, Safra I, Menif S, Abbes S. Genetic polymorphisms of NQO1, CYP1A1 and TPMT and susceptibility to acute lymphoblastic leukemia in a Tunisian population. Mol Biol Rep 2013; 2: 1307-14.

25. Zheng B, Wang Z, Chai R. NQO1 C609T polymorphism and colorectal cancer susceptibility: a meta-analysis. Arch Med Sci 2014; 10: 651-60.

26. Li C, Zhou Y. Association between NQO1 C609T polymorphism and acute lymphoblastic leukemia risk: evidence from an updated meta-analysis based on 17 case-control studies. J Cancer Res Clin Oncol 2014; 6: 873-81.

27. John SW, Weitzner G, Rozen R, Scriver CR. A rapid procedure for extracting genomic DNA from leukocytes. Nucleic Acids Res 1991; 2: 408.

28. Kondo S, Sturgis EM, Li F, Wei Q, Li G. GSTM1 and GSTT1 null polymorphisms and risk of salivary gland carcinoma. Int J Clin Exp Med 2009; 1: 68-75.

29. Jugessur A, Shi M, Gjessing HK, et al. Genetic determinants of facial clefting: analysis of 357 candidate genes using two national cleft studies from Scandinavia. PLoS One 2009; 4: e5385.

30. Purcell S, Neale B, Todd-Brown K, et al. PLINK: a tool set for whole-genome association and population-based linkage analyses. Am J Hum Genet 2007; 3: 559-75.

31. Xu H, Yang W, Perez-Andreu V, et al. Novel susceptibility variants at 10p12.31-12.2 for childhood acute lymphoblastic leukemia in ethnically diverse populations. J Natl Cancer Inst 2013; 10: 733-42.

32. Guo LM, Xi JS, Ma Y, Shao L, Nie CL, Wang GJ. ARID5B gene rs10821936 polymorphism is associated with childhood acute lymphoblastic leukemia: a meta-analysis based on 39,116 subjects. Tumour Biol 2014; 1 709-13.

33. Kennedy AE, Kamdar KY, Lupo PJ, Okcu MF, Scheurer ME, Dorak MT. Genetic markers in a multi-ethnic sample for childhood acute lymphoblastic leukemia risk. Leuk Lymphoma 2015; 56: 169-74.

34. Inaba H, Greaves M, Mullighan CG. Acute lymphoblastic leukaemia. Lancet 2013; 9881: 1943-55.

35. Wang Y, Chen J, Li J, et al. Association of three polymorphisms in ARID5B, IKZF1 and CEBPE with the risk of childhood acute lymphoblastic leukemia in a Chinese population. Gene 2013; 2: 203-7.

36. Vijayakrishnan J, Sherborne AL, Sawangpanich R, Hongeng S, Houlston RS, Pakakasama S. Variation at 7p12.2 and 10q21.2 influences childhood acute lymphoblastic leukemia risk in the Thai population and may contribute 
to racial differences in leukemia incidence. Leuk Lymphoma 2010; 10: 1870-4.

37. Pastorczak A, Gorniak P, Sherborne A, et al. Role of 657 del5 NBN mutation and 7p12.2 (IKZF1), 9p21 (CDKN2A), 10q21.2 (ARID5B) and 14q11.2 (CEBPE) variation and risk of childhood ALL in the Polish population. Leuk Res 2011; 11: 1534-6.

38. Nelis M, Esko T, Magi R, et al. Genetic structure of Europeans: a view from the North-East. PLoS One 2009; 5: e5472.

39. Healy J, Richer C, Bourgey M, Kritikou EA, Sinnett D. Replication analysis confirms the association of ARID5B with childhood B-cell acute lymphoblastic leukemia. Haematologica 2010; 9: 1608-11.

40. Tang ST, Wang CJ, Tang HQ, Zhang Q, Wang Y. Evaluation of glutathione S-transferase genetic variants affecting type 2 diabetes susceptibility: a meta-analysis. Gene 2013; 2: 301-8.

41. Han FF, Guo CL, Gong LL, Jin Z, Liu LH. Effects of the NQ01 609C>T polymorphism on leukemia susceptibility: evidence from a meta-analysis. Asian Pac J Cance Prev 2013; 9: 5311-6. 